\title{
Performance of Spectrum Sensing for Cognitive Radio based on Field Measurements of Various Radio Technologies
}

\author{
Miguel López-Benítez ${ }^{\dagger}$, Fernando Casadevall $^{\dagger}$ and Corrado Martella ${ }^{\ddagger}$ \\ $\dagger$ Department of Signal Theory and Communications, Universitat Politècnica de Catalunya, Barcelona, Spain \\ $\ddagger$ Università di Bologna, Bologna, Italy \\ miguel.lopez@tsc.upc.edu, ferranc@tsc.upc.edu, corrado.martella@ studio.unibo.it
}

\begin{abstract}
Despite its practical performance limitations, energy detection has gained popularity during the last years as a spectrum sensing technique for dynamic spectrum access in cognitive radio networks. The main advantages of energy detectionbased spectrum sensing are its simplicity, low computational and implementation costs as well as its ability to work irrespective of the actual signal to be detected. Since no prior knowledge is required, energy detection can be employed when the secondary receiver cannot gather sufficient information about the primary user signal. Due to the generality of its operating principle, the energy detector performance would not be expected to depend on the type of primary signal being detected. In this context, this paper evaluates the performance of energy detectionbased spectrum sensing for several real-world primary signals of various radio technologies. The obtained results indicate that the detection performances may vary notably with the considered primary signal but converge under certain conditions. The practical consequences of the different observed performances for several primary radio technologies are illustrated and discussed.
\end{abstract}

\section{INTRODUCTION}

One of the most important requisites for a Cognitive Radio (CR) is not cause harmful interference to legitimate primary users. To guarantee interference-free spectrum access, secondary users should reliably identify spectrum opportunities across frequency, time and space [1]. In the context of Dynamic Spectrum Access (DSA), spectrum sensing is traditionally understood as the function in charge of determining which portions of the spectrum are available to secondary users for opportunistic use, which basically means being able to determine whether a primary signal is present within a certain frequency range. This functionality is required not only to detect temporarily unoccupied spectrum bands where secondary users may transmit but also to detect reappearing primary users and trigger evacuation mechanisms in order to avoid harmful interference.

A number of different spectrum sensing methods have been proposed in the literature to identify the presence of primary signal transmissions [2, 3]. They provide different trade-offs between required sensing time, complexity and detection capabilities. Depending on how much information is available about the primary user signal different performances can be reached. However, in the most generic case no prior information is available to a CR user. In such a case energy detection proves an excellent candidate that provides a simple, computationally inexpensive implementation. Energy detection compares the received signal energy in a certain frequency band to a properly set predefined threshold. If the signal lies above the threshold the band is declared to be occupied by the primary network. Otherwise the band is supposed to be idle and could be accessed. Due to the simplicity and generality of this operating principle, the energy detector performance is expected to be independent of the primary signal being detected. In this context, this paper evaluates the performance of energy detection-based spectrum sensing for CR based on various real-world primary signals. The results obtained in this work indicate that the detection performance of energy detection may actually vary with the primary radio technology being considered, but converges under certain conditions. The causes and consequences of the different detection performances for various primary signals are identified, analyzed and discussed.

The rest of this paper is organized as follows. First, section II indicates the main novelties of this work, highlighting the differences with respect to other previous works where the performance of spectrum sensing has been studied. Then, section III presents the considered system model, problem formulation and performance metrics. Afterwards, section IV describes in detail the principles and theoretical results related to energy detection-based spectrum sensing, which is the method considered in this work. The measurement platform and evaluation methodology are detailed in sections V and VI, respectively. Section VII presents and discusses the obtained results, and finally section VIII draws the main conclusions derived from the study reported in this work.

\section{NOVELTIES OF THIS WORK}

Previous works have dealt with the study of spectrum sensing for DSA in CR networks. However, there are a number of aspects that have not been covered satisfactorily in the performance evaluation of spectrum sensing. The following points identify some of such deficiencies and explain how this work fills the existing gaps:

- Spectrum sensing has usually been evaluated by means of theoretical studies or simulations considering simple primary user signals such as sine wave carriers or synthetic $M$-PSK/ $M$-QAM modulated signals [4], which lacks of realism in some practical scenarios. By contrast, this study is performed with real-world signals captured by means of a radio measurement platform.

- In theoretical and simulation studies, primary signals are usually assumed to be affected only by Additive White Gaussian Noise (AWGN), although in practice there exist other noise sources as well as various propagation phenomena impairing the received signal and therefore impacting on the spectrum sensing performance. The performance evaluation of spectrum sensing with real 
signals, as performed in this study, implicitly takes into account all the aspects of the real world that can affect the performance of spectrum sensing algorithms in practice. The need for empirical experiments is stressed by the inability to realistically model all the potential sources of degradation encountered in real receivers and propagation environments.

- Some previous studies have already considered real signals following an approach similar to this study. However, in such cases only TV signals have been considered, and wireless microphone signals in some other cases [5]. Although the first CR deployments are expected in the TV bands following the IEEE 802.22 standard [6], in the future one could expect the deployment of the CR technology in many other interesting bands once the TV bands become crowded. However, very little is known about the performance of spectrum sensing with other primary signals, which might significantly differ from the performance obtained in the case of TV signals. This study evaluates and analyzes the performance of spectrum sensing when considering not only TV signals but also signals from other radio technologies, thus providing a broader view on the performance of spectrum sensing.

In this context, this study experimentally evaluates and comparatively analyzes the performance of spectrum sensing considering real-world primary signals of different radio technologies under a common evaluation framework. This study will allow us not only to corroborate theoretical findings with practical results but also to determine the impact of considering various primary signal technologies on the detection performance of spectrum sensing.

\section{System Model, Problem Formulation AND PERFORMANCE METRICS}

The spectrum sensing problem basically consists in determining if a primary transmission is present in the sensed band. Let us assume that the continuous time Radio Frequency (RF) signal received at the secondary terminal, $y(t)$, can be expressed as:

$$
y(t)=x(t)+w(t)
$$

where $x(t)$ is the primary RF signal to be detected (after passing through a wireless channel including path loss, fading and multipath effects), $w(t)$ is AWGN noise (some other noise sources could be considered) and $t$ is the temporal variable $\left(t \in \mathbb{R}^{+}\right)$. Note that $x(t)=0$ when there is no primary transmission. The received signal $y(t)$ is then shifted to a lower Intermediate Frequency (IF) at which it is sampled at a sampling rate $f_{s}$. Assuming complex bandpass sampling and signal bandwidth $W$, the sampling rate should satisfy $f_{s} \geq W$ if the sensing algorithm needs all the spectral information to be preserved. After sampling, the received discrete signal is:

$$
y[m]=x[m]+w[m]
$$

where $y[m], x[m]$ and $w[m]$ represent, respectively, the discrete time versions of $y(t), x(t)$ and $w(t)$ sampled at instants $t=m T_{s}$, with $m$ being a positive integer $(m \in \mathbb{N})$ representing the sample index and $T_{s}=1 / f_{s}$ is the sampling period.

The received signal is generally passed through a filter. Let $f[k], k=0,1, \ldots, K$, be the filter, where $K$ is the order of the filter. After filtering, the received signal, $\tilde{y}[m]$, becomes:

$$
\tilde{y}[m]=\sum_{k=0}^{K} f[k] \cdot y[m-k]
$$

which can be expressed as

$$
\tilde{y}[m]=\tilde{x}[m]+\tilde{w}[m]
$$

where

$$
\begin{aligned}
\tilde{x}[m] & =\sum_{k=0}^{K} f[k] \cdot x[m-k] \\
\tilde{w}[m] & =\sum_{k=0}^{K} f[k] \cdot w[m-k]
\end{aligned}
$$

If the sampling rate $f_{s}$ is sufficiently larger than the signal bandwidth, it is possible (and convenient in order to reduce the processing load) to down-sample the received signal. Assuming a down-sampling or decimation factor $M \geq 1$, the received signal is:

$$
\tilde{y}[n]=\tilde{x}[n]+\tilde{w}[n]
$$

where $n=M m$.

After properly sampling, filtering and decimating the received signal, the spectrum sensing algorithm has to decide, based on the signal samples $\tilde{y}[n]$, if a primary signal $\tilde{x}[n]$ is present in the received signal or it is only composed of noise samples $\tilde{w}[n]$. Under this approach, the spectrum sensing problem can be formulated as a binary hypothesis testing problem with the following two hypotheses:

$$
\begin{array}{ll}
\mathcal{H}_{0}: \tilde{y}[n]=\tilde{w}[n] & n=0,1, \ldots, N-1 \\
\mathcal{H}_{1}: \tilde{y}[n]=\tilde{x}[n]+\tilde{w}[n] & n=0,1, \ldots, N-1
\end{array}
$$

where $\mathcal{H}_{0}$ is a null hypothesis stating that there is no primary signal in the sensed spectrum band, and hypothesis $\mathcal{H}_{1}$ indicates that some licensed user signal is present. $N$ denotes the number of samples collected during the signal observation interval (i.e., the sensing period), emphasizing that the decision is made based on a limited number of signal samples. Based on this formulation, the spectrum sensing function may be regarded as a black box that accepts a sequence of digital signal samples $\tilde{y}[n]$ as input and provides a binary output indicating whether a primary signal is present in the input sequence $\left(\mathcal{H}_{1}\right)$ or not $\left(\mathcal{H}_{0}\right)$.

The ideal spectrum sensor would select hypothesis $\mathcal{H}_{1}$ whenever a primary signal is present and hypothesis $\mathcal{H}_{0}$ otherwise. Unfortunately, spectrum sensing algorithms may fall into mistakes in practice, which can be classified into miss detections and false alarms. A miss detection occurs when a primary signal is present in the sensed band and the spectrum sensing algorithm selects hypothesis $\mathcal{H}_{0}$, which results in harmful interference to primary users. On the other hand, a false alarm occurs when the sensed spectrum band is free and the spectrum sensing algorithm selects hypothesis $\mathcal{H}_{1}$, which results in missed transmission opportunities and therefore in a lower spectrum utilization. Based on these definitions, the performance of any spectrum sensing algorithm can be summarized by means of two probabilities: the probability of miss detection $P_{m d}=P\left(\mathcal{H}_{0} / \mathcal{H}_{1}\right)$, or its complementary probability of detection $P_{d}=P\left(\mathcal{H}_{1} / \mathcal{H}_{1}\right)=1-P_{m d}$, and the 
probability of false alarm $P_{f a}=P\left(\mathcal{H}_{1} / \mathcal{H}_{0}\right)$. Large $P_{d}$ and low $P_{f a}$ values would be desirable. Nevertheless, there exists a trade-off between $P_{d}$ and $P_{f a}$, meaning that improving one of these performance metrics in general implies degrading the other one and vice versa. In this context, Receiver Operating Characteristic (ROC) curves (obtained by plotting $P_{d}$ versus $\left.P_{f a}\right)$ are very useful since they allow us to explore the relationship between the sensitivity $\left(P_{d}\right)$ and specificity $\left(P_{f a}\right)$ of a spectrum sensing method for a variety of different algorithm parameters and other affecting factors.

\section{ENERGY DETECTION}

A number of spectrum sensing methods have been proposed in the literature to identify the presence of primary signal transmissions $[2,3]$. They provide different trade-offs between required sensing time, complexity and detection capabilities, but their practical applicability depends on how much information is available about the primary user signal. In the most generic case, a CR user is not expected to be provided with any prior information about the primary signals that may be present within a certain frequency range. When the secondary receiver cannot gather sufficient information about the primary user signal, the energy detection principle can be employed due to its ability to work irrespective of the actual signal to be detected. Due to its simplicity and relevance, energy detection has been a preferred approach for many past spectrum sensing studies and also constitutes the approach adopted in this study.

An energy detector, also referred to as radiometer, simply measures the energy received on a primary band during an observation interval and declares the band as occupied if the measured energy is greater than a properly set threshold, or unoccupied otherwise [7]. Thus, the test statistic $\mathbb{T}$ for the energy detector is given by:

$$
\mathbb{T}=\sum_{n=0}^{N-1}|\tilde{y}[n]|^{2}
$$

where $N$ is the size of the observation vector (number of samples collected during the sensing interval). The test statistic $\mathbb{T}$ is compared against a fixed decision threshold $\lambda$ to distinguish between the two hypothesis in equation 8:

$$
\mathbb{T} \underset{\mathcal{H}_{0}}{\stackrel{\mathcal{H}_{1}}{\gtrless}} \lambda
$$

The decision threshold could be chosen for an optimum trade-off between $P_{d}$ and $P_{f a}$. However, this would require knowledge of noise and detected signal powers. While the noise power can be estimated, the signal power is difficult to estimate since it depends on many varying factors such as transmission and propagation characteristics. In practice, the threshold is normally chosen to satisfy a certain $P_{f a}$ [8], which only requires the noise power to be known in order to select a threshold. Unfortunately, calibration errors as well as changes in thermal noise caused by temperature variations limit the accuracy with which noise power can be estimated, leading to some noise uncertainty. Such uncertainty imposes fundamental limitations on detection performance. Although classical detection theory states that degradation in the detection performance due to reduced Signal-to-Noise Ratio (SNR) can be countered by increasing the sensing time $[9,10]$, in practice there exists a limit, referred to as $S N R$ wall, below which the primary signal cannot be reliably detected, regardless of the sensing period [11]. More specifically, modeling the noise uncertainty as $\sigma_{\tilde{w}}^{2} \in\left[\sigma_{\tilde{w}, \text { nominal }}^{2}, \alpha \cdot \sigma_{\tilde{w}, \text { nominal }}^{2}\right]$ [12] $\left(\sigma_{\tilde{w}}^{2}\right.$ is the noise variance), the SNR wall $\gamma_{\text {wall }}$ of the energy detector for a noise uncertainty of $10 \log _{10} \alpha \mathrm{dB}$ is:

$$
\gamma_{\text {wall }}(d B)=10 \log _{10}(\alpha-1)
$$

The existence of SNR walls has been experimentally verified $[4,13]$, demonstrating that a small noise power estimation error may cause significant performance loss in the energy detector.

Closed-form expressions for detection $\left(P_{d}\right)$ and false alarm $\left(P_{f a}\right)$ probabilities can be obtained based on the statistics of $\mathbb{T}$ [14]. The AWGN noise can be modeled as a zero-mean Gaussian random variable. Assuming that the primary signal varies slowly with respect to the noise, the primary signal can be considered to be quasi-deterministic, although unknown in detail. The assumption of a (quasi-)deterministic signal means that the input with a primary signal present is Gaussian but not zero-mean [7]. Then, the test statistic $\mathbb{T}$ follows a central chi-square distribution with $2 N$ degrees of freedom under hypothesis $\mathcal{H}_{0}$, and a non-central chi-square distribution with variance $\sigma^{2}=1$, non-centrality parameter $\mu=2 \gamma$ and $2 N$ degrees of freedom under hypothesis $\mathcal{H}_{1}$ [7]:

$$
\mathbb{T} \sim \begin{cases}\chi_{2 N}^{2}, & \mathcal{H}_{0} \\ \chi_{2 N}^{2}(2 \gamma), & \mathcal{H}_{1}\end{cases}
$$

where $\gamma=\sigma_{\tilde{x}}^{2} / \sigma_{\tilde{w}}^{2}$ is the SNR, and $\sigma_{\tilde{x}}^{2}, \sigma_{\tilde{w}}^{2}$ are the signal and noise variances respectively.

If only AWGN noise is considered, $P_{d}$ and $P_{f a}$ can be evaluated based on the statistics of $\mathbb{T}$ as follows [14]:

$$
\begin{aligned}
P_{d} & =P\left(\mathbb{T}>\lambda \mid \mathcal{H}_{1}\right)=Q_{N}(\sqrt{2 \gamma}, \sqrt{\lambda}) \\
P_{f a} & =P\left(\mathbb{T}>\lambda \mid \mathcal{H}_{0}\right)=\frac{\Gamma(N, \lambda / 2)}{\Gamma(N)}
\end{aligned}
$$

where $\Gamma(\cdot)$ and $\Gamma(\cdot, \cdot)$ are the complete and incomplete gamma functions [15], and $Q_{N}(\cdot, \cdot)$ is the generalized Marcum $Q$ function [16].

The expressions of $P_{d}$ and $P_{f a}$ can be simplified assuming that the observation length $N$ is large. This assumption is frequently valid since the study of spectrum sensing in the context of CR focuses in the low SNR regime (virtually, signal detection under high SNR values does not pose a challenging problem). In low SNR regime the number of required samples $N$ to achieve a certain performance is large. Thus, if the observation length $N$ is long enough, the central limit theorem can be employed to approximate the test statistic as Gaussian:

$$
\mathbb{T} \sim \begin{cases}\mathcal{N}\left(N \sigma_{\tilde{w}}^{2}, 2 N \sigma_{\tilde{w}}^{4}\right), & \mathcal{H}_{0} \\ \mathcal{N}\left(N\left(\sigma_{\tilde{x}}^{2}+\sigma_{\tilde{w}}^{2}\right), 2 N\left(\sigma_{\tilde{x}}^{2}+\sigma_{\tilde{w}}^{2}\right)^{2}\right), & \mathcal{H}_{1}\end{cases}
$$

Under such assumption, $P_{d}$ and $P_{f a}$ are then readily obtained to be:

$$
\begin{aligned}
P_{d} & =Q\left(\frac{\lambda-N\left(\sigma_{\tilde{x}}^{2}+\sigma_{\tilde{w}}^{2}\right)}{\sqrt{2 N\left(\sigma_{\tilde{x}}^{2}+\sigma_{\tilde{w}}^{2}\right)^{2}}}\right) \\
P_{f a} & =Q\left(\frac{\lambda-N \sigma_{\tilde{w}}^{2}}{\sqrt{2 N \sigma_{\tilde{w}}^{4}}}\right)
\end{aligned}
$$


where $Q(\cdot)$ is the standard Gaussian tail probability $Q$ function. Notice that for a desired $P_{f a}$ the threshold $\lambda$ can be set without the knowledge of the signal power:

$$
\lambda=Q^{-1}\left(P_{f a}\right) \sqrt{2 N \sigma_{\tilde{w}}^{4}}+N \sigma_{\tilde{w}}^{2}
$$

Then, for a fixed number of samples $N$ (sometimes constrained by physical layer features or higher layer protocols), $P_{d}$ can be evaluated by substituting $\lambda$ in 16. Each threshold corresponds to a pair $\left(P_{d}, P_{f a}\right)$ in the ROC. If the number of samples $N$ is not limited, then any desired pair $\left(P_{d}, P_{f a}\right)$ can be met simultaneously. For a certain target performance, the minimum number of required samples is:

$$
N=2\left[\frac{Q^{-1}\left(P_{f a}\right)-Q^{-1}\left(P_{d}\right)(1+\gamma)}{\gamma}\right]^{2}
$$

where $\gamma=\sigma_{\tilde{x}}^{2} / \sigma_{\tilde{w}}^{2}$ is the SNR. It is worth noting that under low SNR regime $(\gamma \ll 1)$, the number of samples needed to meet a specified pair $\left(P_{d}, P_{f a}\right)$ scales as $O\left(1 / S N R^{2}\right)$, indicating a relatively high sample complexity of the energy detector.

\section{Measurement Platform}

The measurement platform employed in this study is based on the Universal Software Radio Peripheral (USRP) [17] and GNU Radio [18] architecture. USRP is an openly designed inexpensive Software Designed Radio (SDR) hardware platform that provides radio front-end functionalities, Analogical to Digital and Digital to Analogical Conversion (ADC/DAC), decimation/interpolation with filtering and a Universal Serial Bus 2 (USB2) interface to connect to an off-the-shell Personal Computer (PC). The PC runs the GNU Radio software, a free and open source toolkit that provides a library of signal processing blocks for building SDRs. In addition, it also provides blocks for communicating with the USRP. The general scheme of the measurement platform is illustrated in figure 1, where the notation introduced in section III is included.

The primary signal of interest is captured with an omnidirectional discone-type antenna AOR DN753 that covers the frequency range 75-3000 MHz. The USRP Radio Frequency (RF) front-ends are provided in form of daughter boards that can be plugged to the USRP main board. In this study we have employed two receiver-only daughter boards: TVRX (50$860 \mathrm{MHz}, 8 \mathrm{~dB}$ typical noise figure) and DBSRX (800-2400 $\mathrm{MHz}$, 3-5 dB typical noise figure). The daughter boards are employed to tune to the carrier frequency of the desired primary signal and perform down-conversion to the Intermediate Frequency (IF) at which the USRP main board operates. The USRP main board includes 12-bit ADCs working at $64 \cdot 10^{6}$ samples per second to digitize the received signal and a Field Programmable Gate Array (FPGA) to perform filtering and digital down-conversion (decimation) from the IF band to the Base Band (BB). Decimation is required in order to adapt the incoming data rate to the USB2 and PC computing capabilities. A USB controller sends the digital signal samples to the PC in 16-bit I and 16-bit Q complex data format (4 bytes per complex sample), resulting in a maximum rate of $8 \cdot 10^{6}$ complex samples per second. The maximum RF bandwidth that can be handled is therefore $8 \mathrm{MHz}$ (narrower bandwidths can be selected by adjusting the decimation rate). The host PC runs the GNU Radio's usrp_rx_cfile.py script, which simply collects the digital signal samples sent by the USRP board through the USB2 interface and saves the received BB digital signal sequence to a file in the host PC's hard drive. After the on-line data capturing process, the set of files created by the script are processed off-line. To this end, the energy detection sensing scheme has been implemented in Matlab according the operating principles and theoretical relations that were described in section IV.

\section{Vi. Evaluation Methodology}

Since the aim of this work is to conduct a performance evaluation study of spectrum sensing based on real-world primary signals, signal captures are therefore required. To collect digital signal samples of real signals, the measurement platform described in section $\mathrm{V}$ is employed. To this end, the whole measurement platform was placed on a building roof in urban Barcelona (latitude: $41^{\circ} 23$ ' 20" north; longitude: $2^{\circ}$ 6' 43" east; altitude: 175 meters) with direct line-of-sight to several transmitters located a few tens or hundreds of meters away from the antenna and without buildings blocking the radio propagation. This measurement scenario enabled us to reliably capture the desired signals under high SNR conditions.

With the aid of a spectrum analyzer, various channels were selected for different radio technologies (see table I). Our criterion was to select those channels with the highest observed power levels (in order to maximize the receiving SNR) provided that the activity pattern was constant (i.e., the signal was always present in order to simplify some computations in the off-line data post-processing phase). For broadcast services (analogical TV, digital TV and DAB-T), the selection was straightforward since such kind of transmitters are always active an transmit at high power levels. For UMTS, the activity factor was not an issue (UMTS base stations transmit broadcast information in continuous mode), but the received signal level was carefully observed, selecting channels from the closest base stations (only a few tenths of meters apart). On the other hand, for TETRA, GSM and DCS, the most problematic aspect was the activity factor. In the case of TETRA we were able to identify transmissions from base stations in Downlink-Continuous Transmission (D-CT) mode [19]. In the case of GSM and DCS, we selected various broadcast channels, which could readily be identified with a spectrum analyzer by their high power level.

After having identified the channels to be measured, the required measurements were performed in the above-mentioned location, tuning the measurement platform to the center frequency of each channel ( $f_{\text {center }}$ in table I) and employing the indicated decimation rates and RF gain factors. Decimation rates were selected so that the effectively sampled signal BandWidth (BW) was equal to or greater than the actual signal $\mathrm{BW}$, whereas the gain factor was chosen so as to maximize the received signal level (and hence the receiving SNR) without incurring in saturation. For most of the channels the optimum gain value was $70 \mathrm{~dB}$ and in the particular case of TV (both analogical and digital) the gain was drastically reduced due to the proximity of the TV station $(\approx 3 \mathrm{~km})$.

For each channel, a sequence of $12 \cdot 10^{6}$ samples was captured and the first $2 \cdot 10^{6}$ samples were discarded in order to remove any potential transient peaks that may appear during the first capturing instants of the USRP board. The resulting sequence was filtered in software with Matlab using a highorder digital Butterworth filter with no more than $0.1 \mathrm{~dB}$ of losses in the passband and at least $50 \mathrm{~dB}$ of attenuation in the stopband. The normalized cut-off frequencies for each 

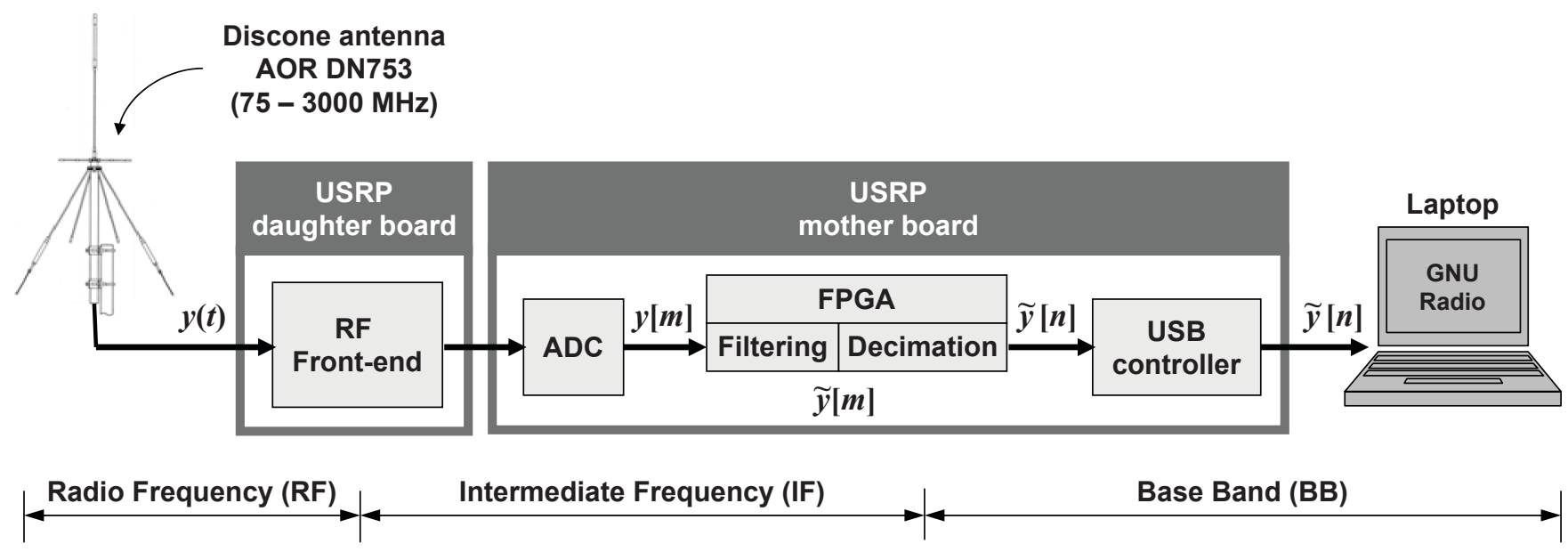

Fig. 1. Measurement platform employed in this study.

TABLE I

Channels measured in this study: AnAlogical/Digital TV, TERREstrial Trunked RAdio (TETRA), TERRESTRial Digital Audio Broadcasting (DAB-T), EXTENDEd GLOBAL SySTEM FOR MOBILE COMMUNICATIONS 900 DOWNLINK (E-GSM 900 DL), Digital CELLULAR SYSTEM 1800 DOWNLINK (DCS 1800 DL) AND UNIVERSAL MOBILE TELECOMMUNICATIONS SYSTEM FREQUENCY-DIVISION DUPLEX DOWNLINK (UMTS FDD DL).

\begin{tabular}{|c|c|c|c|c|c|c|c|c|c|c|}
\hline System & $\begin{array}{l}\text { Channel } \\
\text { number }\end{array}$ & $\begin{array}{l}f_{\text {start }} \\
(\mathrm{MHz})\end{array}$ & $\begin{array}{c}f_{\text {center }} \\
(\mathrm{MHz})\end{array}$ & $\begin{array}{c}f_{\text {stop }} \\
\text { (MHz) }\end{array}$ & $\begin{array}{c}\text { Signal BW } \\
(\mathrm{MHz})\end{array}$ & $\begin{array}{l}\text { Decimation } \\
\text { rate }(M)\end{array}$ & $\begin{array}{l}\text { Sampled BW } \\
(\mathrm{MHz})\end{array}$ & $\begin{array}{l}\text { Gain } \\
(\mathrm{dB})\end{array}$ & $\begin{array}{l}\text { Cut-off } \\
\text { frequency }\end{array}$ & $\begin{array}{c}\text { Pass band } \\
(\mathrm{MHz})\end{array}$ \\
\hline Analogical TV & $\begin{array}{l}23 \\
29 \\
34 \\
38 \\
\end{array}$ & $\begin{array}{l}486 \\
534 \\
574 \\
606 \\
\end{array}$ & $\begin{array}{l}490 \\
538 \\
578 \\
610\end{array}$ & $\begin{array}{l}494 \\
542 \\
582 \\
614\end{array}$ & 8 & 8 & 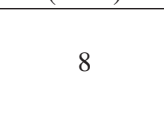 & 10 & 0.94 & 7.52 \\
\hline Digital TV & $\begin{array}{l}26 \\
48 \\
61 \\
67\end{array}$ & $\begin{array}{l}510 \\
686 \\
790 \\
838\end{array}$ & $\begin{array}{l}514 \\
690 \\
794 \\
842\end{array}$ & $\begin{array}{l}518 \\
694 \\
798 \\
846\end{array}$ & 8 & 8 & 8 & 10 & 0.94 & 7.52 \\
\hline TETRA & $\begin{array}{l}37 \\
44 \\
45 \\
47 \\
53\end{array}$ & $\begin{array}{l}420.8875 \\
421.0625 \\
421.0875 \\
421.1375 \\
421.2875\end{array}$ & $\begin{array}{l}420.900 \\
421.075 \\
421.100 \\
421.150 \\
421.300\end{array}$ & $\begin{array}{l}420.9125 \\
421.0875 \\
421.1125 \\
421.1625 \\
421.3125\end{array}$ & 0.025 & 256 & 0.25 & 70 & 0.1 & 0.03 \\
\hline DAB-T & $\begin{array}{l}08 \mathrm{~A} \\
10 \mathrm{~A} \\
11 \mathrm{~B}\end{array}$ & $\begin{array}{l}195.080 \\
209.080 \\
217.784\end{array}$ & $\begin{array}{l}195.936 \\
209.936 \\
218.640\end{array}$ & $\begin{array}{l}196.792 \\
210.792 \\
219.496\end{array}$ & 1.712 & 32 & 2 & 70 & 0.8 & 1.6 \\
\hline E-GSM 900 DL & $\begin{array}{c}60 \\
113 \\
975\end{array}$ & $\begin{array}{l}946.8 \\
957.4 \\
925.0\end{array}$ & $\begin{array}{l}947.0 \\
957.6 \\
925.2\end{array}$ & $\begin{array}{l}947.2 \\
957.8 \\
925.4\end{array}$ & 0.2 & 64 & 1 & 70 & 0.3 & 0.3 \\
\hline DCS $1800 \mathrm{DL}$ & $\begin{array}{l}546 \\
771 \\
786\end{array}$ & $\begin{array}{l}1811.8 \\
1856.8 \\
1859.8\end{array}$ & $\begin{array}{l}1812.0 \\
1857.0 \\
1860.0\end{array}$ & $\begin{array}{l}1812.2 \\
1857.2 \\
1860.2\end{array}$ & 0.2 & 64 & 1 & 70 & 0.3 & 0.3 \\
\hline UMTS FDD DL & $\begin{array}{l}10588 \\
10663 \\
10738\end{array}$ & $\begin{array}{l}2115.1 \\
2130.1 \\
2145.1\end{array}$ & $\begin{array}{l}2117.6 \\
2132.6 \\
2147.6\end{array}$ & $\begin{array}{l}2120.1 \\
2135.1 \\
2150.1\end{array}$ & 5 & 8 & 8 & 70 & 0.625 & 5 \\
\hline
\end{tabular}

channel are shown in table I, resulting in passbands equal to or greater than the signal BW, except for TV channels where some BW was required to accommodate the filter's transient band (for DAB-T the RF bandwidth is $1.712 \mathrm{MHz}$ but the signal information is confined within a $\mathrm{BW}$ of $1.54 \mathrm{MHz}$ ).

Before employing the signals obtained after the previous steps in our experimental study, we verified that they satisfied the two established requirements, namely high signal levels without saturation and activity factors equal to $100 \%$. The fulfillment of the first requisite can be verified from figure 2, where the average power spectrum of some captured signals is shown as an example. As it can be appreciated, the nearly perfect spectral shapes indicate a good SNR level without distortion by saturation. To verify the accomplishment of the second requirement, the antenna shown in figure 1 was replaced with a matched load and the measurements were repeated in order to measure the receiver system's noise. By comparing the instantaneous energy levels of both the signal and noise sequences, we verified that the energy levels present in the signal sequences were significantly higher than those measured for the noise sequences along the whole measurement period, thus indicating that a primary user signal was present with an activity factor of $100 \%$ in all the captured signal sequences.

To compute the performance metrics $P_{d}$ and $P_{f a}$, input sequences are divided into blocks of $N$ samples (sample length) and a sufficiently high number of such blocks is processed. For lower values of $N$, a higher number of blocks is processed in 

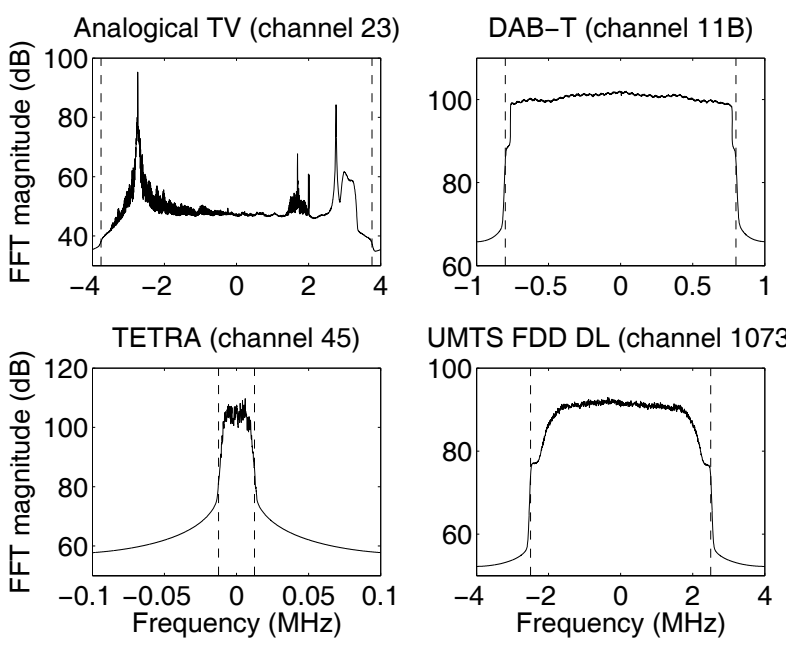

UMTS FDD DL (channel 10738)

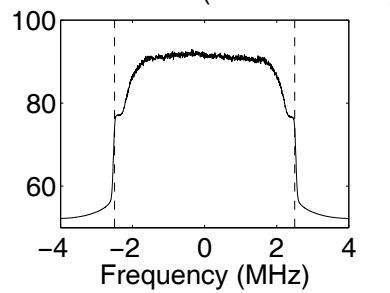

Fig. 2. Average power spectrum (averaged over more than 4800 2048-point FFTs) for some of the captured signals. Dashed lines represent the filter's cut-off frequencies.

order to keep the statistical accuracy of the obtained results at an acceptable level. Assuming that the signal sequence is noise-free (which is a reasonable approximation for high SNR levels), the captured sequence's power is measured and the obtained value is considered as the actual signal power $\sigma_{\tilde{x}}^{2}$. Based on the desired SNR $\gamma$, an AWGN sequence with power level $\sigma_{\tilde{w}}^{2}=\sigma_{\tilde{x}}^{2} / \gamma$ is generated. Based on the values of $N$ and $\sigma_{\tilde{w}}^{2}$, the energy decision threshold for a given target $P_{f a}$ is set according to equation 18, and the energy of each $N$-sample input block is compared to the threshold. To compute the $P_{d}$, the sequence obtained by adding the signal and AWGN sequences is employed as input to the energy detector. The experimental $P_{d}$ is then obtained as the proportion of blocks where the detection result is $\mathcal{H}_{1}$ (the primary signal is present in all the processed blocks since the activity factor for all channels is $100 \%$ ). Similarly, the experimental $P_{f a}$ is computed as the proportion of blocks where the detection result is $\mathcal{H}_{1}$ when the input signal is the AWGN sequence (no primary signal is present in any block).

\section{RESUlTS}

Employing the measurement platform presented in section $\mathrm{V}$ and following the methodology described in section VI, the performance of energy detection-based spectrum sensing has been evaluated for the 25 channels shown in table I. The obtained results indicated similarities among channels of the same radio technology. Since channels of the same system did not manifest significant differences among them in most of the cases, the set of channels corresponding to the same radio technology were averaged in order to obtain a single performance curve for each radio technology. This simplifies the study of the obtained results since only one performance curve needs to be analyzed for each system (instead of one curve per individual channel). The obtained results are shown in figures 3 and 4 in terms of the $P_{d}$ as a function of the SNR. Figure 3 shows the results for the case of perfectly calibrated noise, i.e. no noise uncertainty $(\alpha=0 \mathrm{~dB})$, while figure 4 corresponds to the case where noise uncertainty is present $(\alpha=1 \mathrm{~dB}$ and $\alpha=2 \mathrm{~dB})$.

\section{A. Validation}

As it can be appreciated in figures 3 and 4 , different performance curves are obtained for different systems although the same detection method (energy detection) is employed for all of them. One may think that this phenomenon might be due to the randomness of the experiments, which are based on measurements performed on an number of arbitrary channels during a short measurement period (from 1.25 to 40 seconds depending on the sampling rate, see table I). In fact, any empirical study suffers from a certain random component that cannot be avoided. Concerning this issue, it is worth noting that some experiments were performed with a lower number of iterations than the ones employed to obtain the results shown in this paper. To determine the randomness of such curves and validate the obtained results, the number of iterations was doubled, leading to the curves shown in figures 3 and 4 . Doubling the number of iterations resulted in slightly smoother curves, but it did not change the positions of the curves in the graph, thus guaranteeing the statistical reliability of the obtained results. Moreover, it is worth highlighting, as mentioned above, that the performance curves obtained for different channels of the same system do not manifest significant differences among them. Therefore, the differences observed among the curves for different systems have to be ascribed to the particular features of each radio technology.

For the rest, the obtained results agree with the theoretical predictions. For example, the detection probability $P_{d}$, as expected, decreases with the SNR. On the other hand, increasing the target probability of false alarm $P_{f a}$ implies reducing the energy decision threshold, which in turn results in an improved detection of weak signals and hence an enhanced $P_{d}$. The existing trade-off between $P_{d}$ and $P_{f a}$ can be clearly appreciated by comparing figures 3(a) and 3(b). Moreover, figure 3 indicates that for perfectly calibrated noise the energy detector is a robust detection method, i.e. any arbitrary pair $\left(P_{d}, P_{f a}\right)$ can be met by simply increasing the sample length as indicated in equation 19 . In the presence of noise uncertainty, however, there exists an SNR threshold (the SNR wall) below which the energy detector becomes non-robust, meaning that $P_{m d}=1-P_{d}$ and $P_{f a}$ cannot simultaneously be made to go to zero, no matter how long the observation interval $N$ is. This trend is clearly appreciated in figure 4 , where increasing the observation interval $N$ improves $P_{d}$ only for SNR values above the SNR wall. Below the threshold imposed by the SNR wall, the probability of detection approaches zero regardless of the sensing interval $N$, making signal detection impossible. As observed in figure 4, the existence of the SNR wall is experimentally verified with a very good agreement with the theoretical SNR wall predicted by equation 11 .

\section{B. Analysis}

The results shown in figures 3 and 4 indicate that the performance of the energy detector may strongly depend on the considered primary radio technology. This behavior is not predicted by the classical theoretical results associated to the energy detection method, which were presented in section IV. In fact, for a given set of target $P_{f a}$, sample length $N$ and SNR $\gamma=\sigma_{\tilde{x}}^{2} / \sigma_{\tilde{w}}^{2}$, equations 16,17 and 18 suggest that the resulting performance in terms of $P_{d}$ is unique. However, figures 3 and 4 clearly demonstrate that for constant $P_{f a}$, $N$ and $\gamma$ values, the resulting $P_{d}$ may strongly depend on the primary radio technology being detected. For example, 


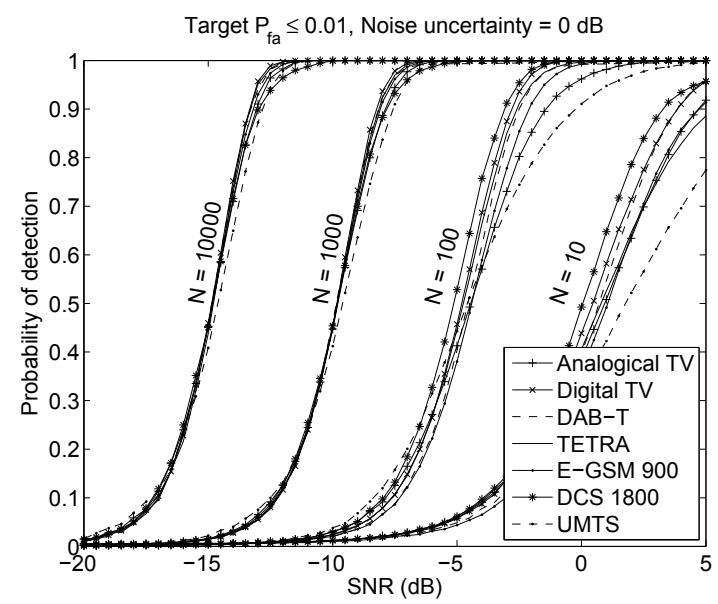

(a)

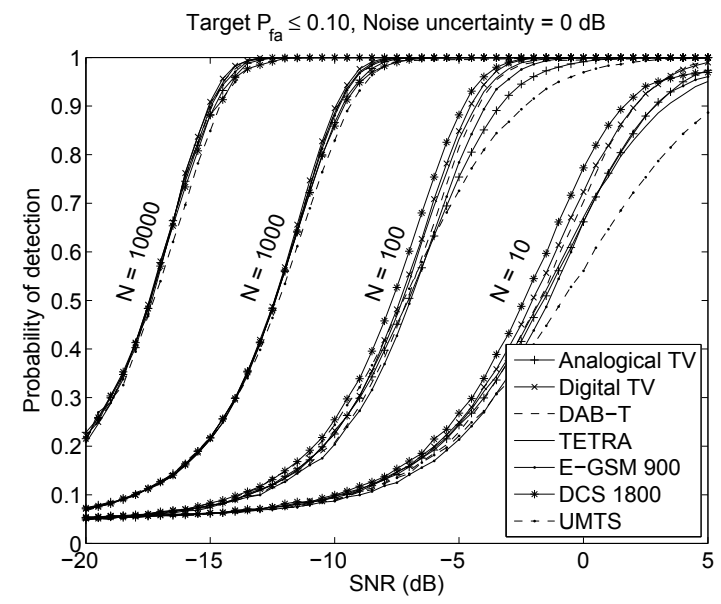

(b)

Fig. 3. Probability of detection versus SNR: (a) $\alpha=0 \mathrm{~dB}$ (target $P_{f a} \leq 0.01$ ), (b) $\alpha=0 \mathrm{~dB}$ (target $P_{f a} \leq 0.10$ ).

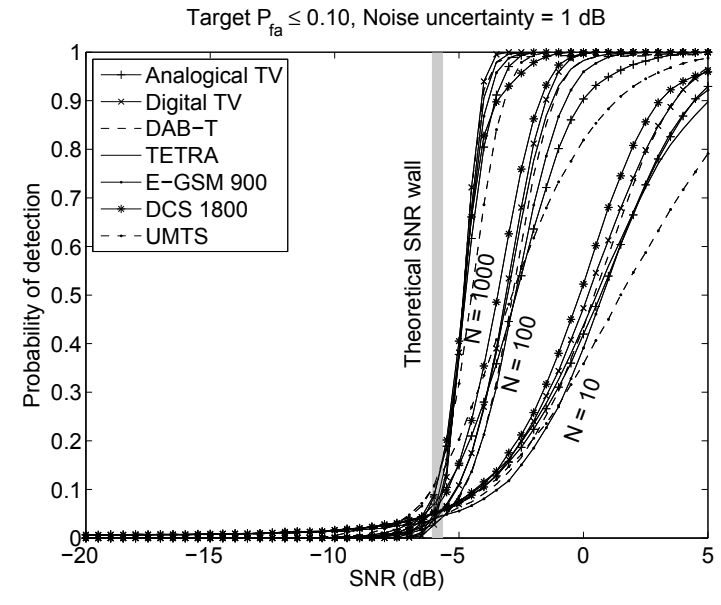

(a)

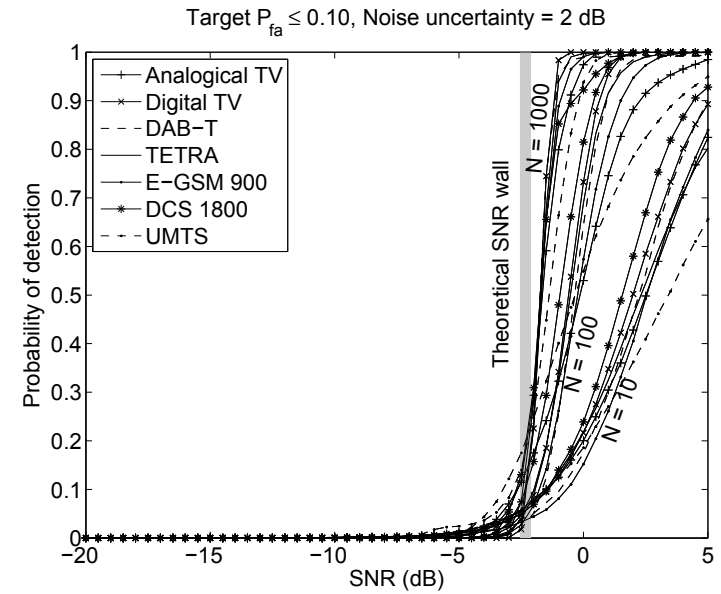

(b)

Fig. 4. Probability of detection versus SNR: (a) $\alpha=1 \mathrm{~dB}$ (target $P_{f a} \leq 0.10$ ), (b) $\alpha=2 \mathrm{~dB}$ (target $P_{f a} \leq 0.10$ ).

for $P_{f a}=0.10, N=10$ and $\gamma=0 \mathrm{~dB}$, equations 16,17 and 18 provide a theoretical detection probability $P_{d}=0.68$. However, for the same parameters, figure 3(b) indicates that the experimental detection probabilities for e.g. digital TV and UMTS are equal to 0.72 and 0.56 , respectively. Moreover, it is interesting to note, as shown in figures 3 and 4, that the performance differences among various radio technologies are not constant, but increase as the sensing period $N$ decreases. This indicates that the sensing period required for a given target performance $\left(P_{d}, P_{f a}\right)$ as predicted by equation 19 , might be enough to detect some primary radio technologies but might actually result insufficient for some others, thus requiring longer sensing periods. If the maximum sensing period is constrained (by physical layer features or higher layer protocols), the resulting detection probability might be acceptable for some radio technologies but might result insufficient to reliably detect other primary signals, thus making some radio technologies more susceptible to interferences under the same conditions. As mentioned in section VII-A, the performance differences observed among various radio technologies have to be ascribed to the particular features of each radio technology. The objective of this section is to identify and analyze the causes that originate the observed differences among various primary signals.

The performance of any spectrum sensing algorithm depends on the decisions made by such algorithm, which in turn depend on the algorithm's test statistic. Since the energy detector's test statistic is related to the received signal energy (see equation 9), it seems reasonable to look for the origins of the observed behavior in the characteristics of the received signal energy or, alternatively, the received signal power. Since the signal power $\mathcal{P}_{\tilde{y}}$ is related to the signal energy $\mathcal{E}_{\tilde{y}}$ by a scaling factor $N$,

$$
\mathcal{P}_{\tilde{y}}=\frac{1}{N} \mathcal{E}_{\tilde{y}}=\frac{1}{N} \sum_{n=0}^{N-1}|\tilde{y}[n]|^{2}
$$


the analysis of the power temporal evolution indicates how the energy detector's test statistic $\mathbb{T}$ varies along time. Analyzing the time evolution of the received signal power is expected to provide some insights into the divergences observed among various radio technologies in figures 3 and 4 .

To evaluate the time evolution of the received signal power, and in order to enable a fair comparison among various signals under the same conditions, the captured sequences $\tilde{y}[n]$ are normalized in order to obtain zero-mean, unit-variance (unitpower) sequences $\check{y}[n]$ as follows:

$$
\check{y}[n]=\frac{\tilde{y}[n]-\mu_{\tilde{y}}}{\sqrt{\sigma_{\tilde{y}}^{2}}}
$$

where $\mu_{\tilde{y}}$ and $\sigma_{\tilde{y}}^{2}$ represent the sample mean and sample variance of sequence $\tilde{y}[n]$, respectively. This operation converts the originally captured sequences $\tilde{y}[n]$ with different absolute signal powers into normalized sequences $\check{y}[n]$ with the same normalized signal power (which in the presence of the same noise power is equivalent to compare them under the same average SNR). The obtained sequences $\check{y}[n]$ are divided into blocks of $N$ samples and the average power of each block is computed. The sequence of received power levels is smoothed by means of a moving average.

The resulting time evolution of the normalized received power is shown in figure 5 for various channels when $N=$ 100. Since such signals were captured under perfect line-ofsight and high SNR conditions, it is reasonable to assume that fading effects are negligible and the observed power variations are therefore mainly due to varying transmission power patterns. In fact, the observed patterns can be related to distinctive signal properties. For example, the highest peaks observed for analogical TV occur every 1600 blocks $\times 100$ samples/block $=1.6 \cdot 10^{5}$ samples, which for the employed sampling rate corresponds to the $50-\mathrm{Hz}$ vertical frequency of the PAL system. The UMTS signal variations can be explained by the use of power control techniques that modify the transmitted power according to the system's load factor. On the other hand, the signals received in digital TV and DCS 1800 (broadcast) channels show a uniform power pattern, as expected.

From figure 5 it is clear that for the selected short observation interval $N=100$, the energy detector's test statistic $\mathbb{T}$ follows the instantaneous variations of the received signal power. In such a case, if the instantaneous signal power falls below the energy decision threshold, the detection result will be $\mathcal{H}_{0}$, even if it should be $\mathcal{H}_{1}$ due to an average energy (power) actually greater than the decision threshold. Therefore, under the same average SNR conditions (i.e., signals with the same average power), this means that a higher power variability implies a higher probability that the instantaneous power level (and the energy detector's test statistic) falls below the decision threshold, which results in a lower detection probability. As a matter of fact, comparing the results shown in figures 3 and 4 for $N=100$ to the power patterns shown in figure 5 , it can be confirmed that the best detection performance is obtained for DCS 1800, followed by digital TV (the signals with the most uniform power patterns), while the worst detection performance corresponds to UMTS FDD, followed by analogical TV (the signals with the most alternating power patterns). This explains the different detection probabilities observed in figures 3 and 4 for various primary signals under

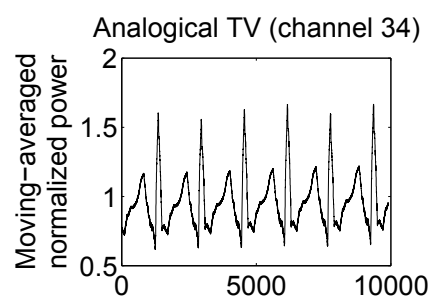

UMTS FDD DL (channel 10738)

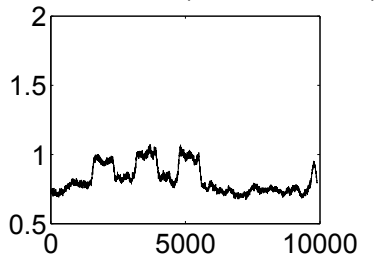

Digital TV (channel 61)

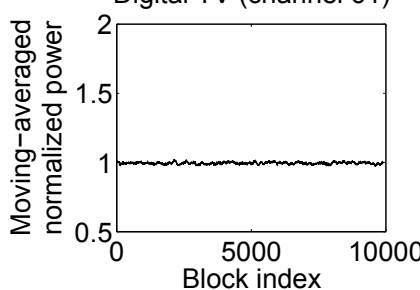

DCS 1800 DL (channel 771)

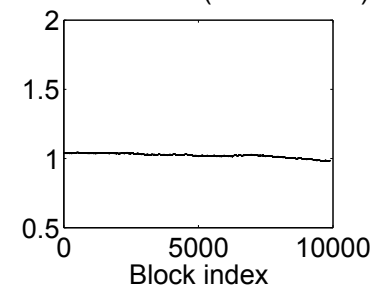

Fig. 5. Moving-averaged normalized power received for some captured signals (moving-averaging window of 100 samples).

the same average SNR conditions. As the number of samples $N$ increases, the test statistic $\mathbb{T}$ is computed over larger observation intervals, thus averaging any instantaneous power variations (the analogue of figure 5 for larger values of $N$ is not shown due to the lack of space, but it ratifies this statement). In such a case, although the variability of the received power remains the same, the variability of the test statistic decreases an so does the probability of miss detection. For sufficiently long observation intervals, the test statistic ceases to follow the instantaneous signal power variations and its value closely resembles the true mean energy (power). When this occurs for all the considered signals, the obtained performance curves converge. i.e. the energy detector performance does not depend on the primary technology considered. This explains the behavior observed in figures 3 and 4 as the value of $N$ increases.

The previous analysis indicates that the energy detector's test statistic may follow the instantaneous variations of the received signal power if short observation intervals are selected. When this occurs, the energy detector's performance strongly depends on the primary technology considered, being degraded for signals with higher power variabilities and affected to a lesser extent when detecting more uniform signal patterns. On the other hand, as the sensing time increases with respect to the primary signal dynamics, the peculiarities of each signal power variation pattern are averaged and the energy detectors's performance becomes therefore more independent of the primary signal being detected.

\section{Discussion}

In spite of the general operating principle of the energy detection method, the results shown in the previous section highlight that certain technology-dependent inherent properties may result in different detection performances for various radio technologies. To quantitatively illustrate the impact of this phenomenon in practice, figure 6 shows the detection probability as a function of the sample length for various primary signals. As it can be appreciated, under the ideal case of no noise uncertainty (upper graph), the performance for 


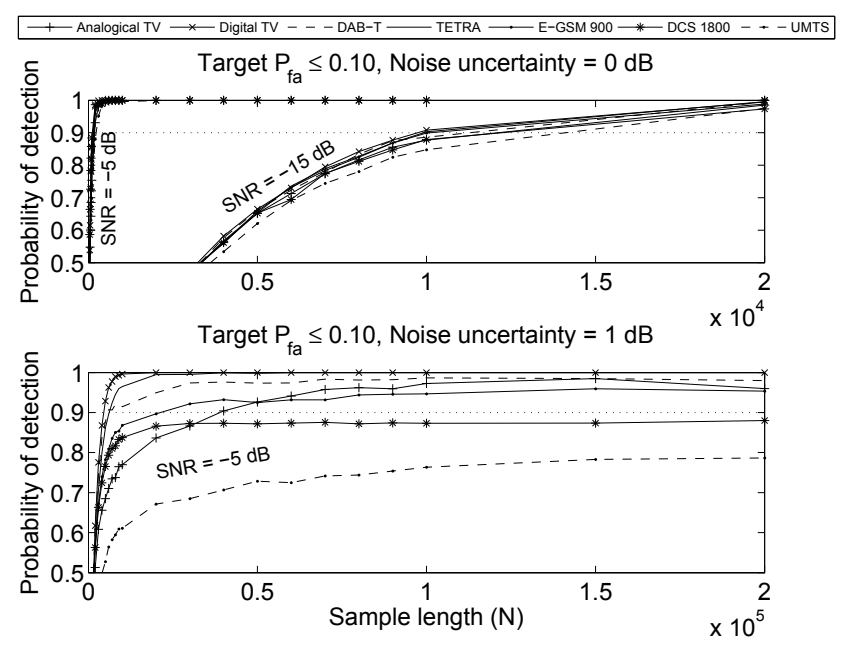

Fig. 6. Probability of detection versus sample length.

different signals follow a similar trend. For example, assuming an average SNR of $-15 \mathrm{~dB}$, a target $P_{d}=0.9$ would require 9745 samples $(\approx 1.22 \mathrm{~ms})$ in the best case (digital TV) and 14099 samples $(\approx 1.76 \mathrm{~ms})$ in the worst case (UMTS). This difference is not very significant, but relies on the assumption that the noise power is perfectly known, which is never true in reality. In the more realistic case of noise uncertainty (lower graph), assuming an average SNR above the SNR wall of -5 $\mathrm{dB}$, a target $P_{d}=0.9$ would require 4532 samples $(\approx 0.57$ $\mathrm{ms})$ in the best case (digital TV), 38937 samples $(\approx 4.87 \mathrm{~ms})$ in an average case (analogical TV), whereas in the worst case (UMTS) the desired $P_{d}$ would never be achieved due to the presence of the SNR wall ( $P_{d}$ for UMTS does not increase beyond 0.8 , no matter how long the observation interval is). These results exemplify the various detection performances that can be reached for different primary radio technologies in reality.

While section VII-B shown that different performances may be obtained with energy detection depending on the primary signal being detected, the results shown in this section indicate that, in practice, such differences can be so important that some primary signals might be reliably detected while some others might not, no matter how long the sensing interval is, thus making some radio technologies more susceptible to interferences under the same conditions.

\section{CONCLUSIONS}

In spite of the general operating principle of the energy detection method, the results shown in this work have indicated that certain technology-dependent inherent properties may result in different detection performances for various primary radio technologies. The detection performance differences are more noticeable for short sensing intervals, where the energy detector's performance is more degraded for signals with higher power variabilities and affected to a lesser extent when detecting more uniform signal patterns. As the sensing interval increases, the energy detectors's performance becomes more similar for different radio technologies and thus more independent of the primary signal being detected. Although increasing the sensing interval improves the detection performance, the existing differences can be so important that, in practice, some primary signals might be reliably detected while some others might not, no matter how long the sensing interval is, thus making some radio technologies more susceptible to interferences under the same conditions. These results indicate that the energy detection principle in the real world may exhibit significantly divergent detection performances depending on the primary signal under detection.

\section{ACKNOWLEDGEMENTS}

This work was supported by the European Commission in the framework of the FP7 Network of Excellence in Wireless COMmunications NEWCOM++ (contract no. 216715). The support from the Spanish Ministry of Science and Innovation (MICINN) under FPU grant AP2006-848 is hereby acknowledged.

\section{REFERENCES}

[1] A. Ghasemi and E. S. Sousa, "Spectrum sensing in cognitive radio networks: requirements, challenges and design trade-offs," IEEE Communications Magazine, vol. 46, no. 4, pp. 32-39, 2008.

[2] T. Yücek and H. Arslan, "A survey of spectrum sensing algorithms for cognitive radio applications," IEEE Communications Surveys and Tutorials, vol. 11, no. 1, pp. 116-130, 2009.

[3] D. D. Ariananda, M. K. Lakshmanan, and H. Nikookar, "A survey on spectrum sensing techniques for cognitive radio," in Proc. of the Second International Workshop on Cognitive Radio and Advanced Spectrum Management (CogART 2009), May 2009, pp. 74-79.

[4] D. Cabric, A. Tkachenko, and R. W. Brodersen, "Experimental study of spectrum sensing based on energy detection and network cooperation," in Proc. of the First International Workshop on Technology and Policy for Accessing Spectrum (TAPAS 2006), Aug. 2006, pp. 1-8.

[5] S. W. Oh, A. A. S. Naveen, Y. Zeng, V. P. Kumar, T. P. C. Le, K. J. M. Kua, and W. Zhang, "White-space sensing device for detecting vacant channels in TV bands," in Proc. of the 3rd International Conference on Cognitive Radio Oriented Wireless Networks and Communications (CrownCom 2008), May 2008, pp. 1-6.

[6] C. Cordeiro, K. Challapali, D. Birru, and N. S. Shankar, "IEEE 802.22: The first worldwide wireless standard based on cognitive radios," in Proc. of the First IEEE International Symposium on New Frontiers in Dynamic Spectrum Access Networks (DySPAN 2005), Nov. 2005, pp. 328-337.

[7] H. Urkowitz, "Energy detection of unknown deterministic signals," Proceedings of the IEEE, vol. 55, no. 4, pp. 523-531, Apr. 1967.

[8] J. J. Lehtomaki, M. Juntti, H. Saarnisaari, and S. Koivu, "Threshold setting strategies for a quantized total power radiometer," IEEE Signal Processing Letters, vol. 12, no. 11, pp. 796-799, Nov. 2005.

[9] S. M. Kay, Fundamentals of statistical signal processing: detection theory. Prentice Hall, 1998, vol. 2.

[10] H. V. Poor, An introduction to signal detection and estimation, 2nd ed. Springer, 1998, vol. 2.

[11] R. Tandra and A. Sahai, "SNR walls for signal detection," IEEE Journal of Selected Topics in Signal Processing, vol. 2, no. 1, pp. 4-17, Feb. 2008.

[12] A. Sahai, N. Hoven, and R. Tandra, "Some fundamental limits on cognitive radio," in Proc. of the Forty-second Allerton Conference on Communications, Control, and Computing (Allerton Conference 2004), Sep. 2004, pp. 1-10.

[13] D. Cabric, A. Tkachenko, and R. W. Brodersen, "Spectrum sensing measurements of pilot, energy, and collaborative detection," in Proc. of the IEEE Military Communications Conference (MILCOM 2006), Oct. 2006, pp. 1-7.

[14] F. F. Digham, M.-S. Alouini, and M. K. Simon, "On the energy detection of unknown signals over fading channels," IEEE Transactions on Communications, vol. 55, no. 1, pp. 21-24, Jan. 2007.

[15] I. S. Gradshteyn and I. M. Ryzhik, Table of integrals, series, and products, 6th ed. Academic, 2000.

[16] A. H. Nuttall, "Some integrals involving the $Q_{M}$-function," IEEE Transactions on Information Theory, vol. 21, no. 1, pp. 95-96, Jan. 1975.

[17] Ettus Research LLC, "Universal Software Radio Peripheral (USRP)," Available at: http://www.ettus.com.

[18] E. Blossom, "Exploring GNU Radio," November 2004, Available at: http://www.gnu.org/software/gnuradio/doc/exploring-gnuradio.html.

[19] ETSI EN 300 392-2, v2.5.2, "Terrestrial Trunked Radio; Voice plus Data; Part 2: Air Interface," Nov. 2005. 